\title{
Iterative Learning and Fractional Order Control for Complex Systems
}

\author{
Farah Bouakrif $\mathbb{D}^{1},{ }^{1}$ Ahmad Taher Azar $\mathbb{D},{ }^{2,3}$ Christos K. Volos $\mathbb{D}$, \\ Jesus M. Muñoz-Pacheco $₫{ }^{5}$, and Viet-Thanh Pham $\mathbb{1}^{6,7}$ \\ ${ }^{1}$ Faculty of Science and Technology, University of Jijel, Jijel 18000, Algeria \\ ${ }^{2}$ College of Engineering, Prince Sultan University, Riyadh, Saudi Arabia \\ ${ }^{3}$ Faculty of Computers and Information, Benha University, Benha 13511, Egypt \\ ${ }^{4}$ Laboratory of Nonlinear Systems-Circuits \& Complexity (LaNSCom), Department of Physics, Aristotle University of Thessaloniki, \\ 54124 Thessaloniki, Greece \\ ${ }^{5}$ Facultad de Ciencias de la Electrónica, Autonomous University of Puebla (BUAP), Av. San Claudio y 18 Sur, Puebla, \\ Pue. 72570, Mexico \\ ${ }^{6}$ Faculty of Electrical and Electronic Engineering, Phenikaa Institute for Advanced Study (PIAS), Phenikaa University, Yen Nghia, \\ Ha Dong district, Hanoi 100000, Vietnam \\ ${ }^{7}$ Phenikaa Research and Technology Institute (PRATI), A\&A Green Phoenix Group, 167 Hoang Ngan, Hanoi 100000, Vietnam
}

Correspondence should be addressed to Farah Bouakrif; f.bouakrif@gmail.com

Received 14 April 2019; Accepted 15 April 2019; Published 2 May 2019

Copyright (c) 2019 Farah Bouakrif et al. This is an open access article distributed under the Creative Commons Attribution License, which permits unrestricted use, distribution, and reproduction in any medium, provided the original work is properly cited.

Control theory asks how to influence the behavior of a dynamical system with appropriately chosen inputs so that the system's output follows the desired trajectory or final state [1-5]. A key notion in control theory is the feedback process: the difference between the actual and desired output is applied as feedback to the system's input, forcing the system's output to converge to the desired output. Feedback control has deep roots in physics and engineering $[6,7]$.

Indeed, when a system is performing the same task repeatedly, it is advantageous to use the knowledge from the previous iterations of the same task to reduce the error on successive trials. An example of such a system is robot arm manipulators when the reference trajectory is repeated over given operation time. When the conventional control algorithms with such systems are used, the same error is repeated from cycle to cycle. Iterative Learning Control (ILC) is a relatively new addition to the toolbox of the control algorithm. It is concerned with the performance of systems that operate repetitively. ILC differs from most existing control methods in the sense that it exploits every possibility of incorporating past control information, such as tracking errors and control input signals, into the construction of the present control action to enable the controlled system to perform progressively better from operation to operation. Since the ILC method was proposed by Uchiyama [8] and presented as a formal theory by Arimoto [9], this technique has been the center of interest of many researchers over the last decades [10-19].

Fractional order control systems have also received considerable attention recently, from both an academic and industrial viewpoint, because of their increased flexibility (concerning integer-order systems) which allows more accurate modeling of complex systems and the achievement of more challenging control requirements [20-24].

The overall purpose of this special issue lies in gathering the latest developments, trends, research solutions, and applications of ILC and fractional order control and to explore the more productive avenues for future research. The breadth of scope for the special issue includes both theoretical study and experimental application. We receive a total of 26 submissions where the authors are from geographically distributed countries, denoting the high impact of the proposed topic and the 
seniority in the organization of the special issue. After two rounds of rigorous review, only ten papers were accepted.

In the paper "Robust Fractional-Order PID Controller Tuning Based on Bode's Optimal Loop Shaping," L. Liu and S. Zhang present a novel fractional-order PID controller tuning strategy based on Bode's optimal loop shaping which is commonly used for LTI feedback systems. The controller parameters are achieved based on flat phase property and Bode's optimal reference model so that the controlled system is robust to gain variations. Also, the proposed fractionalorder PID controller does not have any restriction on the controlled plant so that it can be widely applied to both integer-order and fractional-order systems.

In the paper "A Fractional-Order System with Coexisting Chaotic Attractors and Control Chaos via a Single State Variable Linear Controller," P. Zhou et al. propose a 3D fractionalorder nonlinear system with coexisting chaotic attractors. The fractional-order system exhibits chaotic attractors with the order as low as 2.5431. Meanwhile, a control scheme for the stabilization of the unstable equilibrium is suggested via a single state variable linear controller.

In the paper "New Iterative Method for the Solution of Fractional Damped Burger and Fractional Sharma-TassoOlver Equations," M. J. Khan et al. apply the new iterative method to obtain the approximate solutions of timefractional damped Burger and time-fractional Sharma-TassoOlver equations. The 2nd-order approximate solutions by the new iterative method are in good agreement with the exact solution as compared to the 5th-order solution by the FRDTM.

In the paper "Consensus of Multi-Integral FractionalOrder Multiagent Systems with Nonuniform Time-Delays," J. Liu et al. study the consensus problems for multiintegral fractional-order multiagent systems (MIFOMASs) with nonuniform time-delays. The consensus conditions for MIFOMASs are obtained by a novel frequency-domain method which properly eliminates consensus problems of the systems associated with nonuniform time-delays. Numerical simulations with different parameters demonstrate the results.

In the paper "The Unique Existence of Weak Solution and the Optimal Control for Time-Fractional Third Grade Fluid System," G. Shao et al. study the third grade fluid system with the time-fractional derivative of the order $\propto \in(0,1)$. They first establish a unique existence criterion of weak solutions in the case that the dimension. Then they prove the sufficient condition of optimal pairs.

In the paper "Adaptive Inverse Control Based on Kriging Algorithm and Lyapunov Theory of Crawler Electromechanical System," G. Zhang et al. propose an adaptive inverse control method based on kriging algorithm and Lyapunov theory to improve control accuracy during adaptive driving of the electromechanical system of a crawler. The adaptive travel control law of the crawler was obtained by the Lyapunov theory. Combined with the kriging algorithm, the adaptive driving reverse control method is designed, and the online system is used to update and perfect the inverse system model in real time. The adaptive inverse control method is verified by theoretical analysis and virtual prototype simulation.
In the paper "Complex Dynamics of the Fractional-Order Rössler System and Its Tracking Synchronization Control," $\mathrm{H}$. Wang et al. study the fractional-order Rössler system with a fast discrete iteration using the Adomian decomposition method (ADM) for its implementing on a DSP board. Complex dynamics of the fractional-order chaotic system are analyzed by means of Lyapunov exponent spectra, bifurcation diagrams, and phase diagrams. Moreover, tracking synchronization controllers were theoretically designed and numerically investigated. That lays a foundation for the application of the fractional-order Rössler system.

In the paper "Dynamics Feature and Synchronization of a Robust Fractional-Order Chaotic System," X.-B. Yang et al. introduce a three-dimensional fractional-order chaotic system. The critical finding by analysis is that the position of signal $x_{3}$ descends at the speed of $1 / c$ as the parameter $\mathrm{b}$ increases, and the signal amplitude of $x_{1}, x_{2}$ can be controlled by the parameter $\mathrm{m}$ in terms of the power function with the index $-1 / 2$. Consequently, this system can provide rich encoding keys for chaotic communication. By considering the properties of amplitude and position modulation, the partial projective synchronization and partial phase synchronization are also realized with a linear control scheme. Numerical simulations are executed to confirm the theoretical analysis.

In the paper "Evaluating Fractional PID Control in a Nonlinear MIMO Model of a Hydroelectric Power Station," O. A. Rosas-Jaimes et al. present a fractional PID control for a hydropower plant with six generation units working in an alternation scheme. The parameters and other features of such a set of hydrogeneration units have been used to perform the respective tuning up. To assess the behavior of this controlled system, a model of such nonlinear plant is regulated through a classical PID by classical linearization of its set points, and then a pseudo-derivative part is substituted into a fractional PID. Both sets of resulting signals are compared; the simulations show that the fractional PID has a faster response concerning those plots obtained from the classical PID used.

In the paper "Results on a Novel Piecewise-Linear Memristor-Based Chaotic System," B. Wang studies a kind of piecewise-linear memristor-based chaotic system. Based on a novel and complicated piecewise-linear memristor model, a chaotic system is constructed; then a random sequence extraction approach from the given memristor-based chaotic system is designed. At last the random sequence test is carried out to show the potential application value in encryption field of the new memristor-based chaotic system.

\section{Conflicts of Interest}

The editors declare that they have no conflicts of interest regarding the publication of this special issue.

\section{Acknowledgments}

The guest editorial team would like to thank and express its deep appreciation to all the authors who have submitted 
their works for this special issue. The editors are also grateful to all anonymous reviewers for their rigorous reviews and insightful comments. Additionally, we would like to thank the journal's Editorial Board for being very encouraging and accommodative regarding this special issue. We hope the readers will find this special issue interesting and informative.

Farah Bouakrif Ahmad Taher Azar

Christos K. Volos

Jesus M. Muñoz-Pacheco

Viet-Thanh Pham

\section{References}

[1] A. T. Azar and S. Vaidyanathan, Advances in Chaos Theory and Intelligent Control, vol. 337 of Studies in Fuzziness and Soft Computing, Springer, Germany, 2016.

[2] F. Bouakrif, "Trajectory tracking control using velocity observer and disturbances observer for uncertain robot manipulators without tachometers," Meccanica, vol. 52, no. 4-5, pp. 861-875, 2017.

[3] K. C. Draa, H. Voos, M. Alma, A. Zemouche, and M. Darouach, "LMI-based trajectory tracking for a class of nonlinear systems with application to an anaerobic digestion process," in Proceedings of the 2018 Annual American Control Conference (ACC), pp. 4393-4397, IEEE, Milwaukee, WI, USA, June 2018.

[4] R. A. Garcia and C. E. D’Attellis, "Trajectory tracking in nonlinear systems via nonlinear reduced-order observers," International Journal of Control, vol. 62, no. 3, pp. 685-715, 1995.

[5] X. Zhang, X. Huang, and H. Lu, "Forwarding-based trajectory tracking control for nonlinear systems with bounded unknown disturbances," International Journal of Control, Automation, and Systems, vol. 14, no. 5, pp. 1231-1243, 2016.

[6] A. T. Azar and S. Vaidyanathan, Chaos Modeling and Control Systems Design, vol. 581 of Studies in Computational Intelligence, Springer, Germany, 2015.

[7] A. T. Azar and Q. Zhu, Advances and Applications in Sliding Mode Control systems, vol. 576 of Studies in Computational Intelligence, Springer, Germany, 2015.

[8] M. Uchiyama, "Formulation of high-speed motion pattern of a mechanical arm by trial," Transactions of the Society of Instrument and Control Engineers, vol. 14, pp. 706-712, 1978.

[9] S. Arimoto, S. Kawamura, and F. Miyazaki, "Bettering operation of robots by learning," Journal of Robotic Systems, vol. 1, no. 2, pp. 123-140, 1984.

[10] F. Bouakrif, "D-type iterative learning control without resetting condition for robot manipulators," Robotica, vol. 29, no. 7, pp. 975-980, 2011.

[11] F. Bouakrif, D. Boukhetala, and F. Boudjema, "Velocity observer-based iterative learning control for robot manipulators," International Journal of Systems Science, vol. 44, no. 2, pp. 214-222, 2013.

[12] F. Bouakrif and M. Zasadzinski, "High order iterative learning control to solve the trajectory tracking problem for robot manipulators using Lyapunov theory," Transactions of the Institute of Measurement and Control, vol. 40, no. 15, pp. 4105-4114, 2018.

[13] F. Bouakrif, "Iterative learning control for strictly unknown nonlinear systems subject to external disturbances," International Journal of Control, Automation, and Systems, vol. 9, no. 4, pp. 642-648, 2011.
[14] M. M. Ghazaei Ardakani, S. Z. Khong, and B. Bernhardsson, "On the convergence of iterative learning control," Automatica, vol. 78, pp. 266-273, 2017.

[15] J. van Zundert, J. Bolder, and T. Oomen, "Optimality and flexibility in iterative learning control for varying tasks," Automatica, vol. 67, pp. 295-302, 2016.

[16] S. Devasia, "Iterative learning control with time-partitioned update for collaborative output tracking," Automatica, vol. 69, pp. 258-264, 2016.

[17] X. Li and D. Shen, "Two novel iterative learning control schemes for systems with randomly varying trial lengths," Systems \& Control Letters, vol. 107, pp. 9-16, 2017.

[18] D. Meng and K. L. Moore, "Convergence of iterative learning control for SISO nonrepetitive systems subject to iterationdependent uncertainties," Automatica, vol. 79, pp. 167-177, 2017.

[19] R. Zhang, Z. Hou, H. Ji, and C. Yin, "Adaptive iterative learning control for a class of non-linearly parameterised systems with input saturations," International Journal of Systems Science, vol. 47, no. 5, pp. 1084-1094, 2016.

[20] A. T. Azar, A. G. Radwan, and S. Vaidyanathan, Mathematical Techniques of Fractional Order Systems, Elsevier, Amsterdam, Netherlands, 2018.

[21] A. T. Azar, S. Vaidyanathan, and A. Ouannas, Fractional Order Control and Synchronization of Chaotic Systems, vol. 688 of Studies in Computational Intelligence, Springer, Germany, 2017.

[22] Y. Q. Chen, I. Petráš, and D. Y. Xue, "Fractional order control-a tutorial," in Proceedings of the American Control Conference (ACC '09), pp. 1397-1411, IEEE, June 2009.

[23] S. Victor, R. Malti, H. Garnier, and A. Oustaloup, "Parameter and differentiation order estimation in fractional models," Automatica, vol. 49, no. 4, pp. 926-935, 2013.

[24] Z. Yakoub, M. Amairi, M. Chetoui, B. Saidi, and M. Aoun, "Model-free adaptive fractional order control of stable linear time-varying systems," ISA Transactions ${ }^{\circledR}$, vol. 67, pp. 193-207, 2017. 


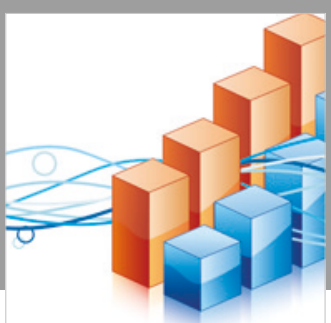

Advances in

Operations Research

\section{-n-m}
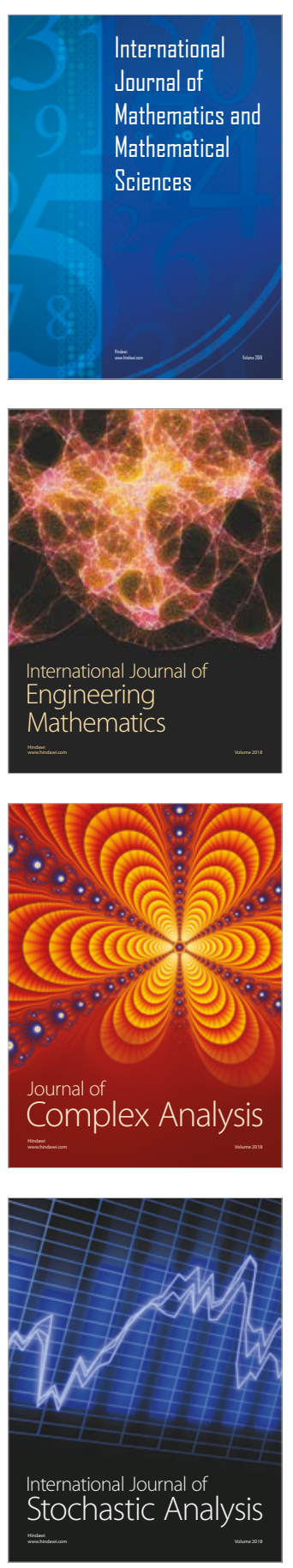
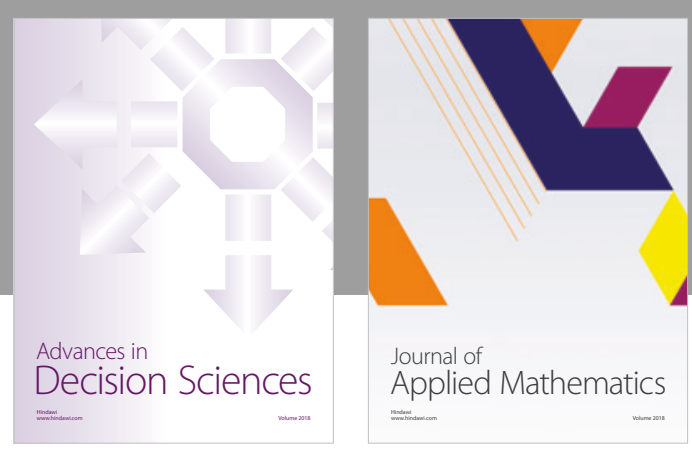

Journal of

Applied Mathematics
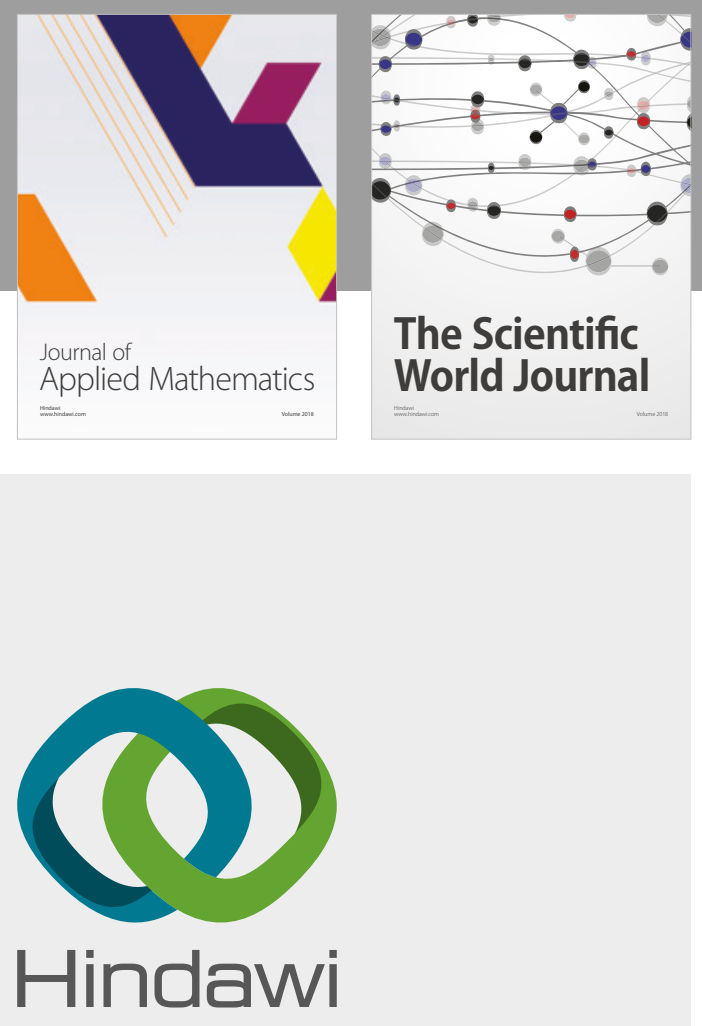

Submit your manuscripts at

www.hindawi.com

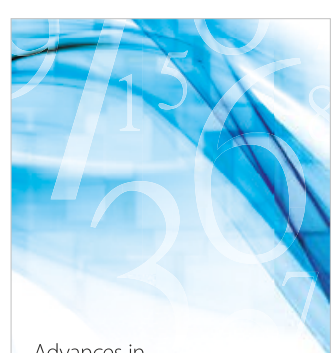

Advances in
Numerical Analysis
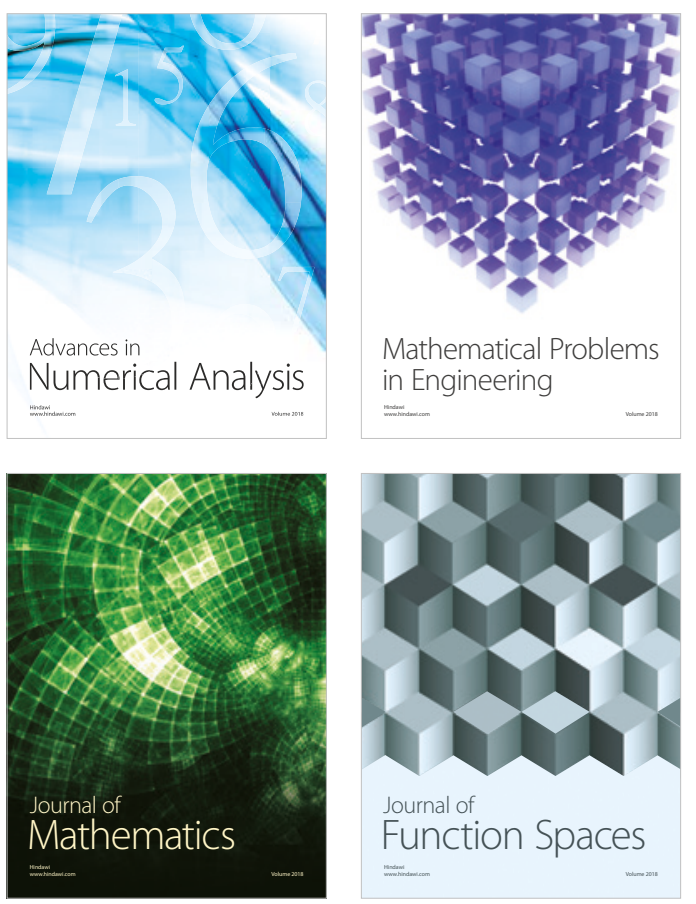

Mathematical Problems in Engineering

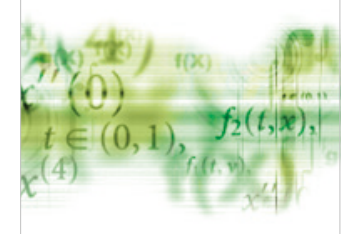

International Journal of

Differential Equations

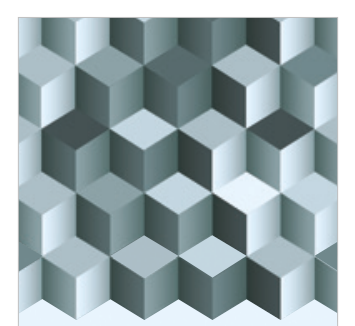

Journal of

Function Spaces
The Scientific

World Journal

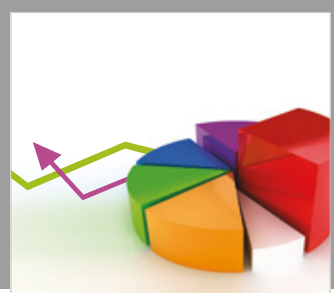

Journal of

Probability and Statistics
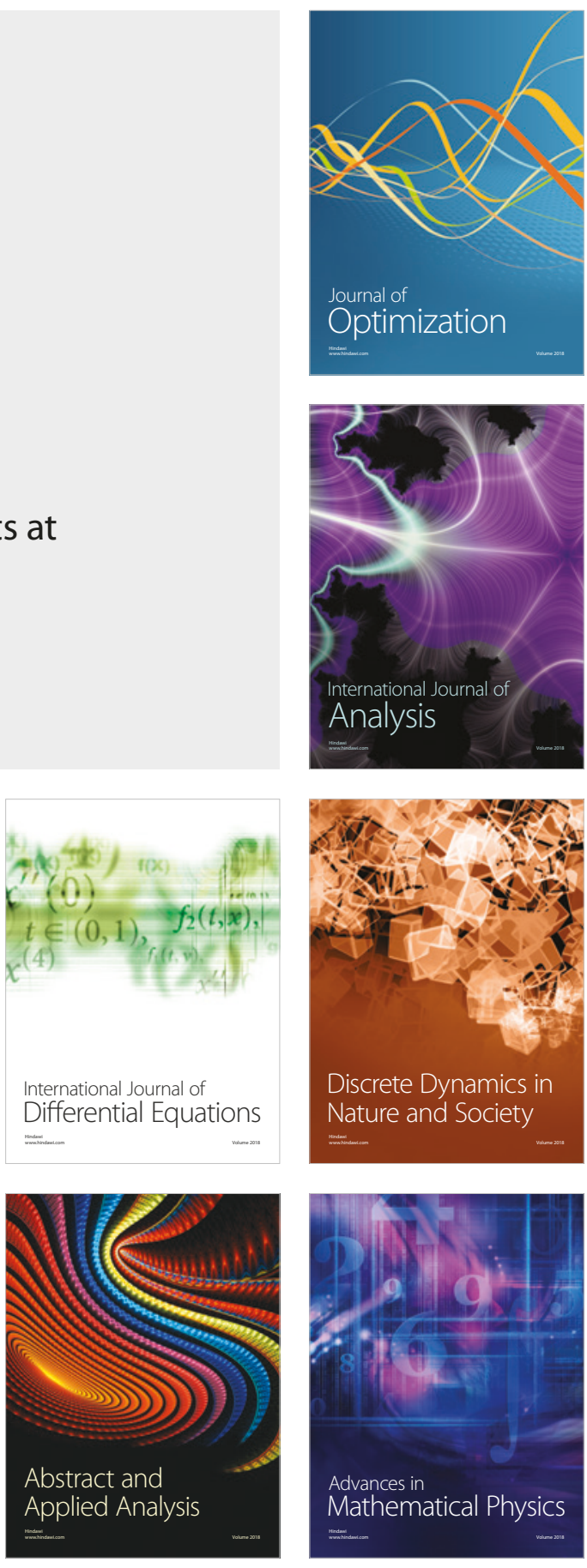